\title{
Reduction of Market Power and Stabilisation of Outcomes in a Novel and Simplified Two-Settlement Electricity Market
}

\author{
Nicolas Höning \\ Centrum Wiskunde en Informatica (CWI) \\ Amsterdam, The Netherlands \\ Email: nicolas@cwi.nl
}

\author{
Han La Poutré \\ Centrum Wiskunde en Informatica (CWI)/Utrecht University \\ Amsterdam/Utrecht, The Netherlands \\ Email: hlp@cwi.nl
}

\begin{abstract}
Modern electricity markets conduct a twosettlement procedure. Ahead of time, they allocate definite supply as well as reserves. Close to the time of consumption, they balance supply and demand. Bidding in these two auctions poses a challenge for automated bidding by agents, which will be more common in future electricity markets and so-called "smart grids". In a decision-theoretic model, we implement the current bidding practice that uses two independent bids and a novel, unified format that simplifies computation. We show through Monte-Carlo simulations in one-shot settings that the unified format restricts market power of suppliers in exploitable settings, and is also less vulnerable to uncertainty of bidders about market outcomes.
\end{abstract}

Keywords-Power System Economics, Electronic Commerce, Smart Grids

\section{INTRODUCTION}

The principal engineering challenge in power systems is to keep costs of production low and to maintain a constant balance between supply and demand. The ongoing deregulation of electricity markets adds the challenge to increase competition in markets with (currently) high concentration of market power at the suppliers. At the same time, both more volatile generation methods (e.g. renewables) as well as more flexible technological components are being introduced, while electricity remains difficult to store. Based on these developments, the "smart grid" concept envisions more dynamic pricing and more decisions made close to the time of consumption. Intelligent agents can potentially take over many planning decisions and place bids in the name of their owners. but for this an advancement in the understanding of the dynamics and proposals for computationally efficient mechanisms are needed.

This work builds on a type of market allocation mechanism that combines ahead-planning with dynamic pricing. The two-settlement procedure, which is currently being used in many deregulated electricity markets, trades a continuous good (electricity) in two different markets. The first settlement clears an "ahead market" (usually one day ahead of time, but shorter intervals are possible in more dynamic market settings, e.g. in "smart grids"). Generators submit a bid to sell some quantity definitively. The ahead market also allocates an additional quantity from generators, but only as an option. The second settlement clears a "balancing market". Generators submit a bid in order to sell some part of this option to even out short-term imbalances.

Specifically, fast-reacting generators like gas power plants or batteries will become more valuable, in order to act as reserve power in the balancing market. It is, however, not settled how to allocate quantities and prices for balancing markets. Is the quantity of needed reserves chosen independently from the quantity of definitely allocated power? Should power only be paid for when it is used for balancing or should the market pay for the availability of fast-startable reserve capacity, regardless of whether it is put to use? This paper uses a version of the two-settlement procedure in which the market maker allocates reserve capacity relative to definitely allocated capacity and dynamically sets prices for electricity which is actually used for balancing.

Bidding in the two-settlement procedure is complex and it is not well-understood how intelligent agents would perform. In a decision-theoretic model, we investigate the profit maximisation problem of a generator on an ahead market (that is part of the two-settlement procedure) and its effects on market prices and the market power of the generator. In a parallel companion work [4] to this work, we investigate the suitability of this format for a flexible consumer.

We advance the state of the art in the following ways: First, we extensively model the decision problem for a smart bidding agent (who is able to offer reserve capacity to the balancing market) in the two-settlement model by implementing the current practice, where separate bids are made for definitively sold power and reserve power. Second, we implement a unified bid format that was proposed by the authors in [5] into this model. The format simplifies the optimisation problem of the bidding agent substantially. Finally, we study varying what-if scenarios with stochastic Monte-Carlo simulations. Experimental evidence shows that the combined format is appealing to market makers, as it restricts the exercise of excessive market power by bidders in exploitable market settings (compared to a non-unified approach). It is also appealing to bidders, as it shows a good performance in all other settings and is less vulnerable to increasing uncertainty about market outcomes. 


\section{BACKGROUND}

Modern electricity markets are centralised, two-sided multi-unit auctions, which are challenging to analyse. They have often used uniform-price auctions (UPA) designs, in which all participants pay or earn the same unit price. The last decade has seen more markets designed as discriminatory pricing auctions (DPA)[7], in which unit prices may differ based on individual bids. Generally, UPA settings have been found to result in more efficient allocations, but DPA designs lower prices and market power of suppliers, see for example Fabra et al. (2002) [3] and Damianov et al. (2010) [2]. This paper models a DPA design.

A very common (e.g. [6], [1], [14]) mathematical model of bids in electricity markets are supply functions, based on a quadratic representation of total production costs: $f(Q)=$ $c Q+R Q^{n}$. This makes the assumption that there are no fixed costs. $Q$ denotes a quantity of electricity and $c \in \mathbb{R}$ and $R \in \mathbb{R}$ are coefficients. Also, $c>0, R>0$ and $n>1$.

Market power, defined as "the ability to alter profitably prices away from competitive levels" [9], lies currently mainly with suppliers, who face inelastic demand. To tackle this problem, electricity market designers have been searching for the best trade-off in bid format design, which allows bidders to freely express their economic preferences, but also restricts them artificially in order to limit prices and the exercise of market power. For instance, Baldick (2002) [1] mentions that quadratic terms in the bids could be limited or bids could be required to be consistent across time.

In the two-settlement procedure described above, the market maker procures reserve energy. The required optimal quantity is currently chosen by static heuristics, for example based on the capacity of the largest power plant, by remaining capacity in allocated generation facilities, or on a percentage of peak capacity in the market. Although they are delivered together as an indistinguishable product, definitive and reserve electricity are priced independently. An important question is when to submit the supply bids for each type. Most scientific literature favours simultaneous approaches (submit at the same time) over sequential ones (submit bids for reserve electricity after the market for definitive electricity has been cleared). In sequential approaches, recommitments can lead to inefficient allocations.

However, a problem with simultaneous approaches is that a bid in one market cannot refer to outcomes in the other market. This reduces chances of reaching efficient allocations. Only few proposals to tackle this problem exist. For example, Virag et al. (2011) [15] propose an iterative market design, where in each round the market maker proposes two market prices and the market participants update the quantities they would sell or buy at those prices. This runs until conversion, but the runtime properties of this dynamic method are uncertain. The problem has also been discussed in Höning et al. (2011) [5], where a combined bid format is proposed for the two-settlement procedure which allocates reserve quantities relative to definitely sold quantities. and a preliminary market clearing mechanism was sketched as constraint satisfaction problem. This paper analyses the performance of this bid format as described in Section I, from the viewpoint of a bidder.

\section{MOdEL}

We consider a generator $g$ using a decision-theoretic approach. $g$ aims at maximising profits from selling electricity in the two markets and his task is to construct appropriate bids for this. In this section, we describe the mathematical form of bids, the ahead market $A$, the balancing market $B$ and $g$ 's profit maximisation problem.

\section{A. Mathematical form of bids}

We denote the maximal production capacity of any generator $h$ with $Q_{h}^{U}$ and $h$ 's total cost function by $C_{h}(Q)=$ $c_{h} Q+R_{h} Q^{2}$, where $Q$ is a quantity of electricity. In our market model, bids are supply functions and map unit prices to quantities (to model the current practice, market $B$ also accepts a constant price bid, see Section IV). A bid by generator $h$ is a linear function. It is based on $C_{h}^{\prime-1}$, the inverse derivative of the total cost function $C_{h}$, wherein $h$ can adapt the minimal offer price per unit $c_{h}$.

First, $C_{h}^{\prime-1}$ is derived as follows: Let $C_{h}^{\prime}$ be the derivative of $C_{h}$ and $\rho_{m}$ be the marginal unit cost related to a quantity of power $Q$ (i.e. $\rho_{m}$ equals the costs of the last produced unit). Then, $C_{h}^{\prime}(Q)=c_{h}+2 R_{h} Q=\rho_{m}$. We can invert $C_{h}^{\prime}$ by solving for $Q$, thus $Q=\frac{1}{2 R_{h}}\left(\rho_{m}-c_{h}\right)=C_{h}^{\prime-1}\left(\rho_{m}\right)$. In economic theory, $C_{h}^{\prime-1}$ is called the marginal cost function and represents the profit-maximising bid in a perfectly competitive market [12]. For comparison, the average unit cost function is the inverse of $\frac{C_{h}(Q)}{Q}$, which is $\frac{1}{R_{h}}\left(\rho_{t}-c_{h}\right)$, where $\rho_{t}$ is the average unit cost. Second, $h$ can construct a bid $b_{h}$ in order to maximise profits by deviating from both his $c_{h}$ and $R_{h}$ value. For simplicity, we fix $R_{h}$ and restrict $h$ to adapt only the parameter $c_{h}$. We represent this by: $b_{h}(\rho)=\frac{1}{2 R_{h}}\left(\rho-c_{h}^{*}\right)$, where $\rho$ is a unit price and $c_{h}^{*}$ is the parameter that $h$ can freely choose. In [1], this restriction of the function parametrisation is called "cparametrisation" and previous literature that also used this restriction is described.

\section{B. The two markets}

Let the overall offered quantity be $Q^{U}$. Each generator $h$ submits bids $b_{h}^{A}$ and $b_{h}^{B}$ for both markets simultaneously, before market $A$ is cleared. We call the market maker $S O$ (which is short for System Operator). The $S O$ uses all bids $b_{h}^{A}$ to clear the aggregate demand on market $A$. The $S O$ definitely buys one part of $Q^{U}$, called $Q^{A}$. Another part, called $Q^{o p t}$, is allocated as an option on electricity and determined by the $S O$ alone. Later in market $B$, some part $Q^{B} \in\left[0, Q^{o p t}\right]$ of this option might be bought. When the 
demand side announces their demand in market $B$, all supply bids $b_{h}^{B}$ are used to clear market $B$.

This work models the decision problem of one generator agent $g$. In both markets, we model the residual demand function $D_{g}$ that $g$ effectively faces, given demand and supply from all other market participants. The residual demand is the full market demand minus the quantity supplied by other generators at each unit price $\rho$ [12]. Thus, let $D(\rho) \rightarrow$ $\mathbb{R}$ be an aggregated demand function and $S_{-g}(\rho) \rightarrow \mathbb{R}$ an aggregated supply function, where $S_{-g}$ aggregates all generators besides $g$. Then, $D_{g}(\rho)=D(\rho)-S_{-g}(\rho)$. Following [12], the latter functions are given by:

$$
\begin{aligned}
D(\rho) & =D_{\max }-\alpha \rho \\
S_{-g}(\rho) & =\beta\left(\rho-\rho_{\min }\right)
\end{aligned}
$$

where $D_{\max }$ denotes the maximal demand (if price is zero), $\alpha$ denotes the slope of $D, \rho_{\min }$ denotes the minimal unit offer price and $\beta \in[0,1]$ denotes the slope of $S_{-g} . D_{g}$ is given by:

$$
\begin{aligned}
D_{g}(\rho) & =D(\rho)-S_{-g}(\rho) \\
& =D_{\text {max }}+\beta \rho_{\text {min }}-\rho(\alpha+\beta)
\end{aligned}
$$

Market clearing works as follows: Given $g$ 's bid $b_{g}$, the quantity $Q_{g}$ that $g$ sells and the unit price $\rho_{b}$ that $g$ earns are found at the intersection of residual demand and $g$ 's bid, thus $D_{g}\left(\rho_{b}\right)=b_{g}\left(\rho_{b}\right)$. However, we also need to consider outcomes of this intersection that would lead to invalid quantities (quantities which $g$ is not able to produce). $g$ can not produce negative quantities, so if $D_{g}\left(\rho_{b}\right)<0, g$ sells nothing, as his bid $b_{g}$ was too expensive. Furthermore, we model an individual maximal capacity constraint $Q_{g}^{\max }$ for $b_{g}$ (for $S_{-g}$, we assume that individual capacity constraints of the aggregated suppliers are not exceeded and thus not relevant for the market clearing). $g$ is willing to sell $Q_{g}^{\max }$ at price $\rho_{b, \max }$ (thus, $b_{g}\left(\rho_{b, \max }\right)=Q_{g}^{\max }$ ) and the $S O$ is willing to pay $\rho_{D, \max }$ (thus, $\left.D_{g}\left(\rho_{D, \max }\right)=Q_{g}^{\max }\right)$. If $b_{g}\left(\rho_{b}\right)>Q_{g}^{\max }$ (see Figure 1 for illustration), we use a discriminative (also called pay-as-bid) auction approach [7], such that $g$ will sell $Q_{g}^{\max }$ at a unit price of $\rho_{b, \max }$ if $\rho_{b, \max } \leq \rho_{D, \max }$ or sell nothing otherwise.

From now on, we denote with $Q_{g}^{A}, Q_{g}^{\text {opt }}$ and $Q_{g}^{B}$ the quantities allocated to $g$ and with $\rho_{g}^{A}$ and $\rho_{g}^{B}$ the unit prices that $g$ earns on markets $A$ and $B$, respectively. For $g$ 's competitors, we denote with $Q_{-g}^{A}$ and $Q_{-g}^{B}$ the quantities that they sell and with $\rho_{-g}^{A}$ and $\rho_{-g}^{B}$ the prices they earn.

Markets $A$ and $B$ are coupled as follows: From now on, we will use $D, D_{g}, S_{-g}$ and their parameters with the superscripts ${ }^{A}$ for market $A$ and ${ }^{B}$ for market $B$. If we omit the superscripts, then the setting is equal in both markets. Equations 3 and 4 show $D$ and $S_{-g}$ for markets $A$ and $B$, where $[X]_{\geq 0}$ denotes the maximum of $X$ and 0 .

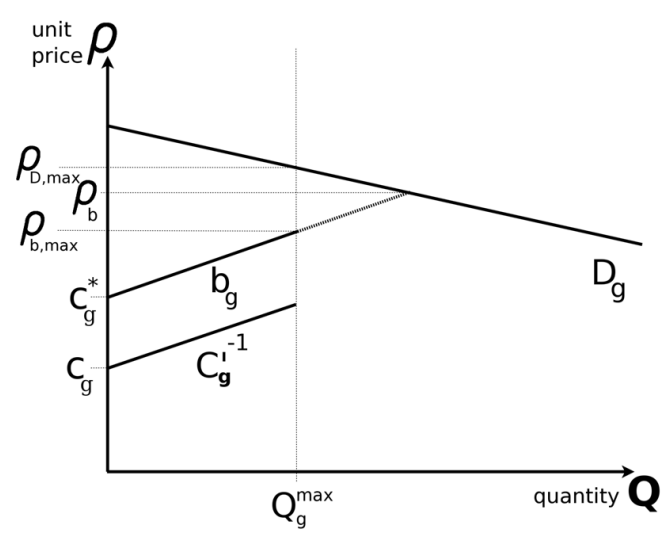

Figure 1. Market clearing. $g$ 's bid $b_{g}$ is constrained by $D_{g}$ and $Q_{g}^{\max }$.

$$
\begin{aligned}
D^{A}(\rho) & :=\left[D_{\text {max }}^{A}-\alpha^{A} \rho\right]_{\geq 0} \\
S_{-g}^{A}(\rho) & :=\left[\beta^{A}\left(\rho-\rho_{\text {min }}^{A}\right)\right]_{\geq 0} \\
D^{B}(\rho) & :=\left[D_{\text {max }}^{B}-\alpha^{B} \rho\right]_{\geq 0} \\
S_{-g}^{B}(\rho) & :=\left[\beta^{B}\left(\rho-\rho_{\text {min }}^{B}\right)\right]_{\geq 0}
\end{aligned}
$$

Two parameters of market $B$ are determined by the outcome of market $A$, while the other two remain freely configurable. First, we assume that the maximal demand in market $B$ is related to $Q^{A}$ via a ratio $r_{m}$, such that $D_{\max }^{B}=\frac{r_{m} Q^{A}}{1-r_{m}}$. The $S O$ can approximate the ratio $r_{m}$ by experience. We assume for simplicity of our mechanism that he approximates $r_{m}$ perfectly and allocates $Q^{o p t}=D_{\max }^{B}$. Furthermore, we assume that he uses $r_{m}$ also for $g$ individually, so it always holds that $Q_{g}^{o p t}=\frac{r_{m} * Q_{g}^{A}}{1-r_{m}}$. We will evaluate scenarios with different values for $r_{m}$ and assume in this work that $g$ as well as (the aggregation of) other generators are able to ramp up enough of their capacity in time for balancing. In addition, we assume that other generators bid the price they achieved in market $A$ as minimum price in market $B$, thus $\rho_{\text {min }}^{B}=\rho_{-g}^{A}$. By this assumption, $\rho_{-g}^{B} \geq \rho_{-g}^{A}$, which is a reasonable expectation, because prices for balancing power are usually higher than for power bought on an ahead market [11]. In our model, there are now six settings free for parametrisation, namely $D_{\text {max }}^{A}, \alpha^{A}, \beta^{A}, \rho_{\text {min }}^{A}, \alpha^{B}$ and $\beta^{B}$.

Finally, we model uncertainty about market outcomes. $g$ knows the residual demand functions $D_{g}^{A}$ and $D_{g}^{B}$. From $g$ 's point of view, $D_{g}^{A}$ and $D_{g}^{B}$ shift up- or downward with an independent motion in markets $A$ and $B$. These shifts are determined by noise parameters $k^{A}$ and $k^{B}$, with which we multiply the minimal price of the competition: 
Table I

SUMMARY OF MARKET PARAMETERS AND MARKET OUTCOMES. WITH THE EXCEPTION OF $r_{m}$ AND $Q_{g}^{o p t}$, WE USE THEM WITH SUPERSCRIPTS ${ }^{A} \mathrm{OR}^{B}$ TO DENOTE THEIR USAGE IN MARKET $A$ OR $B$, RESPECTIVELY.

\begin{tabular}{|c|l|}
\hline Parameter & Description \\
\hline \hline$D_{\max }$ & maximal demand of demand function $D$ \\
\hline$\alpha$ & slope of $D$ \\
\hline$\rho_{\min }$ & min. price of supply function $S_{-g}(g$ 's competition) \\
\hline$\beta$ & slope of $S_{-g}$ \\
\hline$k$ & noise parameter \\
\hline$r_{m}$ & $\begin{array}{l}\text { describes ratio between } Q^{A} \text { and } D_{\max }^{B} \text { as well as } \\
\text { between } Q_{g}^{A} \text { and } Q_{g}^{o p t}\end{array}$ \\
\hline \hline Outcome & Description \\
\hline \hline$Q_{g}, Q_{-g}$ & quantities that $g$ and $g$ 's competition sell \\
\hline$Q_{g}^{o p t}$ & quantity that $g$ holds in reserve for market $B$ \\
\hline$\rho_{g}, \rho_{-g}$ & prices that $g$ and $g$ 's competition earn \\
\hline
\end{tabular}

$$
\begin{aligned}
& S_{-g}^{A}\left(\rho, k^{A}\right)=\beta^{A}\left(\rho-\rho_{\text {min }}^{A} k^{A}\right) \\
& S_{-g}^{B}\left(\rho, k^{B}\right)=\beta^{B}\left(\rho-\rho_{\text {min }}^{B} k^{B}\right)
\end{aligned}
$$

\section{The profit maximisation problem}

$g$ has two bids to construct (we call them $b_{g}^{A}$ and $b_{g}^{B}$ ), one for market $A$ and one for market $B$. We now consider the optimisation of bids in terms of profits for $g$. The profit in each market is the revenues minus the total production costs. In market $A$, revenues are $Q_{g}^{A} * \rho_{g}^{A}$ and the total costs of producing $Q_{g}^{A}$ are given by $C_{g}\left(Q_{g}^{A}\right)$. In market $B$, revenues are $Q_{g}^{B} * \rho_{g}^{B}$. The total costs of producing $Q_{g}^{B}$ are the costs for producing the last $Q_{g}^{B}$ units in $Q_{g}^{A}+Q_{g}^{B}$. Therefore, we introduce a total cost function $C_{g}^{b a l}$ for $Q_{g}^{B}$ that calculates the costs on $C_{g}\left(Q_{g}^{A}+Q_{g}^{B}\right)$ for $\stackrel{Q}{g}_{g}^{B} \in\left[0, Q_{g}^{o p t}\right]$ :

$$
\begin{aligned}
C_{g}^{b a l}\left(Q_{g}^{A}, Q_{g}^{B}\right) & =C_{g}\left(Q_{g}^{A}+Q_{g}^{B}\right)-C_{g}\left(Q_{g}^{A}\right) \\
& =\left(c_{g}+2 R_{g} Q_{g}^{A}\right) Q_{g}^{B}+R_{g}\left(Q_{g}^{B}\right)^{2}
\end{aligned}
$$

Then, the profit functions are:

$$
\begin{aligned}
\operatorname{profits}_{g}^{A}\left(b_{g}^{A}, k^{A}\right) & =\rho_{g}^{A} Q_{g}^{A}-C_{g}\left(Q_{g}^{A}\right) \\
\operatorname{profits}_{g}^{B}\left(b_{g}^{B}, b_{g}^{A}, k^{B}\right) & =\rho_{g}^{B} Q_{g}^{B}-C_{g}^{b a l}\left(Q_{g}^{A}, Q_{g}^{B}\right)
\end{aligned}
$$

where $Q_{g}^{A}$ and $\rho_{g}^{A}$ are determined through market clearing as described in Section III-B, when $g$ submits the bid $b_{g}^{A}$ to market $A$, with $k^{A}$ being the noise variable and $Q_{g}^{U}\left(1-r_{m}\right)$ being $g$ 's maximal capacity in market $A . Q_{g}^{B}$ and $\rho_{g}^{B}$ are likewise determined through market clearing when $g$ submits bid $b_{g}^{B}$ to market $B$, with $k^{B}$ being the noise variable and $Q_{g}^{\text {opt }}=\frac{r_{m} * Q_{g}^{A}}{1-r_{m}}$ being $g$ 's maximal capacity in market $B$. Note that profits $s_{g}^{B}$ is coupled to the results of market $A$ (and thus needs to consider $b_{g}^{A}$ ), as $Q_{g}^{A}$ is used in $C_{g}^{b a l}$ as well as in the determination of $Q_{g}^{o p t}$.

We now formulate the profit maximisation problem for $g . g$ considers limited ranges of noise parameters $k^{A}$ and $k^{B},\left[k_{\text {min }}^{A}, k_{\max }^{A}\right]$ and $\left[k_{\text {min }}^{B}, k_{\text {max }}^{B}\right]$, respectively. For the likelihood of $k^{A}$ and $k^{B}$, let the two probability lookup functions be $\operatorname{prob}^{A}\left(k^{A}\right) \rightarrow[0,1]$ and $\operatorname{prob}^{B}\left(k^{B}\right) \rightarrow[0,1]$. The profit maximisation problem for $b_{g}^{A}$ and $b_{g}^{B}$ is shown below. For each possible outcome for bid $b_{g}^{A}, g$ considers all possible outcomes for bid $b_{g}^{B}$.

$$
\begin{aligned}
& \arg \max _{b_{g}^{A}, b_{g}^{B}}\left[\int _ { k ^ { A } = k _ { \text { min } } ^ { A } } ^ { k _ { \text { max } } ^ { A } } \operatorname { p r o b } ^ { A } ( k ^ { A } ) * \left(\operatorname{profits}^{A}\left(b_{g}^{A}, k^{A}\right)\right.\right. \\
& \left.\left.+\int_{k^{B}=k_{\text {min }}^{B}}^{k_{\text {max }}^{B}} \operatorname{prob}^{B}\left(k^{B}\right) * \operatorname{profits}^{B}\left(b_{g}^{B}, b_{g}^{A}, k^{B}\right) d k^{B}\right) d k^{A}\right]
\end{aligned}
$$

\section{BID FORMATS}

In the reference format (which we call $B E N C H$ ), $g$ submits two independent bids: a bid $b_{g}^{A}$ to market $A$, which is a supply function of the format $b_{g}^{A}(\rho)=\frac{1}{2 R_{g}} *\left(\rho-c_{g}^{*}\right)$, and a bid $b_{g}^{B}$ to market $B$, which is a constant price $\rho_{g}^{B *}$.

In the unified bid format proposed in [5] (which we call $U N I), g$ bids only one bid $b_{g}(\rho)=\frac{1}{2 R_{g}}\left(\rho-c_{g}^{*}\right)$. First, the $S O$ clears market $A$, using $b_{g}$ as $b_{g}^{A}$. Given the outcome of market $A$ ( $g$ sells $Q_{g}^{A}$ at unit price $\rho_{g}^{A}=$ $\left.b_{g}{ }^{-1}\left(Q_{g}^{A}\right)=c_{g}^{*}+2 R_{g} Q_{g}^{A}\right)$, the $S O$ then constructs bid $b_{g}^{B}$ from $b_{g}$ to submit to market $B$. $b_{g}^{B}$ defines $b_{g}\left(\rho_{g}^{B}\right)$ for $\rho_{g}^{B} \in\left[\rho_{g}^{A}, \rho_{b_{g}, \max }\right]$, where $b_{g}\left(\rho_{b_{g}, \max }\right)=Q_{g}^{A}+Q_{g}^{o p t} . b_{g}^{B}$ is based on $b_{g}$, with the minimal unit price $\left(c_{g}^{*}\right)$ replaced by $\rho_{g}^{A}$ (see $b_{g}^{B}(\rho)$ in (9)). The $U N I$ format reduces the complexity of $g$ 's optimisation problem by one dimension to only one bid $b_{g}$ and thus simplifies the computation that a bidding agent has to perform:

$$
\begin{gathered}
\arg \max _{b_{g}}\left[\int _ { k ^ { A } = k _ { \text { min } } ^ { A } } ^ { k _ { \text { max } } ^ { A } } \operatorname { p r o b } ^ { A } ( k ^ { A } ) * \left(\operatorname{profits}_{g}^{A}\left(b_{g}, k^{A}\right)\right.\right. \\
\left.\left.+\int_{k^{B}=k_{\min }^{B}}^{k_{\max }^{B}} \operatorname{prob}^{B}\left(k^{B}\right) * \operatorname{profits}_{g}^{B}\left(b_{g}^{B}, b_{g}, k^{B}\right) d k^{B}\right) d k^{A}\right] \\
\text { where } b_{g}^{B}(\rho)=\frac{1}{2 R}\left(\rho-\left(c_{g}^{*}+2 R_{g} Q_{g}^{A}\right)\right)
\end{gathered}
$$

In contrast to the $B E N C H$ format, the $U N I$ format makes it possible to use the same bidding strategy on market $A$ and $B$. For instance, a marginal cost bid $b_{g}(\rho)=$ $\frac{1}{2 R_{g}}\left(\rho-c_{g}\right)$ also bids marginal unit costs with bid $b_{g}^{B}$.

\section{Simulation EXPERIMENTS}

We construct two market scenarios and investigate several settings in each, using a systematic parameter analysis. In each setting, $g$ constructs bids for markets $A$ and $B$. We then investigate the resulting market outcome via Monte Carlo simulations. To assess the effect of $g$ 's decision on the overall market, we measure an aggregated measure of unit prices over both markets combined, dividing the revenue of all suppliers by all sold power: 


$$
\frac{\rho_{g}^{A} * Q_{g}^{A}+\rho_{-g}^{A} * Q_{-g}^{A}+\rho_{g}^{B} * Q_{g}^{B}+\rho_{-g}^{B} * Q_{-g}^{B}}{Q_{g}^{A}+Q_{-g}^{A}+Q_{g}^{B}+Q_{-g}^{B}}
$$

Furthermore, we want to quantify $g$ 's ability to profitably alter prices away from competitive levels. To this end, economists measure the market power of actors. We measure market power by calculating the Lerner index $\in[0,1]$, defined by dividing per-unit profits by unit price. As the index is defined for a monopolist, we multiply it by $g$ 's market share [13]:

$$
\operatorname{lerner}(Q)=\frac{\rho_{g}(\vec{Q})-\operatorname{costs}_{g}(\vec{Q})}{\rho_{g}(\vec{Q})} s_{m}(\vec{Q})
$$

where $\rho_{g}$ is the average unit price $g$ earns when selling the quantities $\vec{Q}=\left(Q_{g}^{A}, Q_{g}^{B}\right)$, costs $s_{g}$ is the average production costs per unit and $s_{m}$ is the market share. In our case:

$$
\begin{aligned}
\rho_{g}\left(Q_{g}^{A}, Q_{g}^{B}\right) & =\frac{Q_{g}^{A} \rho_{g}^{A}+Q_{g}^{B} \rho_{g}^{B}}{Q_{g}^{A}+Q_{g}^{B}} \\
\operatorname{costs}_{g}\left(Q_{g}^{A}, Q_{g}^{B}\right) & =\frac{C_{g}\left(Q_{g}^{A}+Q_{g}^{B}\right)}{Q_{g}^{A}+Q_{g}^{B}} \\
s_{m}\left(Q_{g}^{A}, Q_{g}^{B}\right) & =\frac{Q_{g}^{A}+Q_{g}^{B}}{Q_{g}^{A}+Q_{-g}^{A}+Q_{-g}^{B}+Q_{g}^{B}}
\end{aligned}
$$

Besides market power and aggregated unit price, we also measure $g$ 's profits, prices $\rho_{g}^{A}, \rho_{g}^{B}$ and quantities $Q_{g}^{A}, Q_{g}^{B}$.

\section{A. Oligopolistic market scenario}

First, we define an oligopolistic market scenario with realistic settings from a wholesale power market simulation study by Sun \& Tesfatsion (2007) [14]. In [14], several generators and a generic buyer profile are described for 24 hours of a day on an electricity wholesale market. In particular, the oligopolistic scenario corresponds to hour 8 in that study (we chose hour 8 as it is similar to most other hours and not an outlier). Because we use settings from a wholesale market study, the prices in our model are in $\$ / M W h$ - but we note that the general findings of this model can also hold for markets which trade $K W h$.

We parametrise $g$ as a typical generator, according to [14]. We set $c_{g}=18.8, R_{g}=0.008$ and $Q_{g}^{U}=300$.

For the parametrisation for $D$ and $S_{-g}$ on the markets $A$ and $B$, Table II lists the default setting for this scenario, which we discuss below, as well as variation ranges.

Furthermore, we run simulations with $r_{m}=0.1$, which is a reserve level often in use today, as well as $r_{m}=0.3$, a setting that is not unrealistic in the market scenarios we can expect in the upcoming 10 years, at least for the generators that can offer significant reserve power.

The sum of the demand of all buyers in [14] is 900, or $3 Q_{g}^{U}$. We set $D_{\max }^{A}=3 Q_{g}^{U}\left(1-r_{m}\right)$. The overall
Table II

DEFAULT SETTINGS AND VARIATION RANGES FOR MARKET PARAMETERS IN THE OLIGOPOLISTIC SCENARIO. THE PARAMETERS FOR MARKET $B$ VARY IN DEPENDENCE TO THE PARAMETERS OF MARKET $A$.

\begin{tabular}{|c|l|l|}
\hline Name & Default setting & Variation range \\
\hline \hline$D_{\max }^{A}$ & $3 Q_{g}^{U}\left(1-r_{m}\right)=810\left[r_{m}: 0.1\right]$ & {$\left[2 Q_{q}^{U}\left(1-r_{m}\right)\right.$,} \\
& $=630\left[r_{m}: 0.3\right]$ & $\left.9 Q_{g}^{U}\left(1-r_{m}\right)\right]$ \\
\hline$\alpha^{A}$ & 1.0 & {$[0.0,5.0]$} \\
\hline$\rho_{\min }^{A}$ & $1.1 c_{g}=20.68$ & {$\left[0.9 c_{g}, 1.5 c_{g}\right]$} \\
\hline$\beta^{A}$ & $\frac{4}{2 R_{g}}=250$ & {$\left[\frac{D_{\max }^{A}}{300}, \frac{D_{\max }^{A}}{15}\right]$} \\
\hline$D_{\max }^{B}$ & $\frac{r_{m} Q^{A}}{1-r_{m}}$ & \\
\hline$\alpha^{B}$ & $\frac{\alpha^{A}}{5}=0.2$ & \\
\hline$\rho_{\min }^{B}$ & $\rho_{-g}^{A}$ & \\
\hline$\beta^{B}$ & $\beta^{A}=250$ & \\
\hline
\end{tabular}

demand in market $B$ depends on the sales of market $A$ (see Section III-B), thus we set $D_{\max }^{B}=\frac{r_{m} Q^{A}}{1-r_{m}}$.

For the slope of the demand functions, we use a survey report [8] of several demand responsiveness studies. All studies in [8] measured the price elasticity of demand, which denotes the percentage change in quantity demanded in response to a one percent change in price. [8] distinguishes between "long-run" and "short-run" demand, where the latter allows less substitution of demanded power by any alternative, similar to the situation in a balancing market. The survey reports price elasticities between 0.7 and 2.1 for "long-run" scenarios (which we use for market $A$ ) and between 0.03 and 0.5 in "short-run" scenarios (which we use for market $B$ ). We take $\alpha^{A}=1.0$ and $\alpha^{B}=0.2=\frac{\alpha^{A}}{5}$ ).

Furthermore, [14] uses five generators in their model. We assume that all generators have the same slope in their production costs. Thus, we multiply the slope of $g$ 's marginal costs by four to get the slope of $S_{-g}: \beta=\frac{4}{2 R_{g}}$. Finally, we assume that the minimal unit price of $S_{-g}$ is $10 \%$ higher than $g$ 's minimal unit costs: $\rho_{\text {min }}^{A}=1.1 c_{g}$. As described in Section III-B, $\rho_{\min }^{B}$ is set to $\rho_{-g}^{A}$.

\section{B. Competitive market scenario}

To provide an outlook into market scenarios of the upcoming 10 years, we also design a scenario with a competitive market (which is more relevant smart grids), see Table III. We base it on the oligopolistic scenario we described in Section V-A. Here, we assume the number of players on the supply side to be ten times higher and set $\beta=\frac{40}{2 R_{g}}$. Furthermore, we also assume that demand responsiveness is ten times higher and set $\alpha^{A}=10$ and $\alpha^{B}=2$. Both assumptions are likely approximations, as the number of suppliers as well as the demand responsiveness are very low in current markets and are expected to increase rapidly in future energy systems. Finally, we assume the overall demand to be twice as high and set $D_{\max }=6 Q_{g}^{U}\left(1-r_{m}\right)$. This assumption is reasonable, because electricity demand is expected to increase, especially with increasing market 
Table III

DEFAULT SETTINGS AND VARIATION RANGES FOR MARKET PARAMETERS IN THE COMPETITIVE SCENARIO.

\begin{tabular}{|c|l|l|}
\hline Name & Default setting & Variation range \\
\hline \hline$D_{\max }^{A}$ & $\begin{array}{r}6 Q_{g}^{U}\left(1-r_{m}\right)=1620\left[r_{m}: 0.1\right] \\
=1260\left[r_{m}: 0.3\right]\end{array}$ & $\begin{array}{c}{\left[4 Q_{g}^{U}\left(1-r_{m}\right),\right.} \\
\left.18 Q_{g}^{U}\left(1-r_{m}\right)\right]\end{array}$ \\
\hline$\alpha^{A}$ & 10 & {$[5.0,15.0]$} \\
\hline$\rho_{\min }^{A}$ & $1.1 c_{g}=20.68$ & {$\left[0.9 c_{g}, 1.5 c_{g}\right]$} \\
\hline$\beta^{A}$ & $\frac{40}{2 R_{g}}=2500$ & {$\left[\frac{D_{\max }^{A}}{300}, \frac{D_{\max }^{A}}{15}\right]$} \\
\hline$D_{\max }^{B}$ & $\frac{r_{m} Q^{A}}{1-r_{m}}$ & \\
\hline$\alpha^{B}$ & $\frac{\alpha^{A}}{5}=2$ & \\
\hline$\rho_{\min }^{B}$ & $\rho_{-g}^{A}$ & \\
\hline$\beta^{B}$ & $\beta^{A}=2500$ & \\
\hline
\end{tabular}

penetration of electric vehicles. Also, the addition of storage facilities to the power systems is both needed and expected.

\section{Method}

In order to systematically create parametrised settings in both scenarios, we vary the values of one parameter at a time while the others remain at the default setting. Per parameter, we select 7 evenly-spaced values from a value range. We vary $r_{m} \in[0.01,0.4], R_{g} \in[0.001,0.03], \phi \in[0.0,3.0]$ and the market parameters $D_{\max }^{A}, \alpha^{A}, \rho_{\min }^{A}$ and $\beta^{A}$ according to the variation intervals indicated in Tables II and III.

For each setting, $g$ constructs either two bids $b_{g}^{A}$ and $b_{g}^{B}$ (with the $B E N C H$ format) or one bid $b_{g}$ (with the $U N I$ format). First, $g$ performs a brute-force search on bid parameter settings: $g$ evaluates 100 evenly-spaced values for $c_{g}^{*} \in\left[c_{g}, \rho_{\max }^{A}\right]$ and, with the $B E N C H$ format, also evaluates for each value of $c_{g}^{*} 100$ evenly-spaced values for $\rho_{g}^{B *} \in\left[c_{g}, \rho_{\max }^{B}\right]$, where $D_{g}^{A}\left(\rho_{\max }^{A}, 1\right)=0$ and $D_{g}^{B}\left(\rho_{\max }^{B}, 1\right)=0$, given $Q_{g}^{A}=0$. Starting with the most promising point from the brute force evaluations, $g$ then applies a downhill simplex algorithm [10] to maximise his expected profits.

We sample the outcomes for each setting 100 times and report the average results together with one standard deviation in our graphs. Each sample uses a new pair of the noise parameters $k^{A}$ and $k^{B}$, generated by the Mersenne twister pseudo-random number generation algorithm. We assume $k^{A}$ and $k^{B}$ to be distributed normally and thus have to choose means and standard deviations in both markets $A$ and $B$. We set the means to 1 and choose each standard deviation $s$ in the following way: We denote $\rho_{\max }$ as the maximal unit price in the market (such that $D_{g}\left(\rho_{\max }, 1\right)=0$ ). We then define $s$ such that $D_{g}\left(\rho_{\text {min }}, 1+\phi s\right)=0$, where $\rho_{\text {min }}$ is the minimal price of $S_{-g}$ (see Section III-B) and $\phi$ is a scaling parameter (in default settings, $\phi=1$ ). The difference of $\rho_{\max }-\rho_{\min }$ is dependent on the market setting and also differs in markets $A$ and $B$. Thus, the noise in the market is proportional to the maximal price variation in residual demand. During the the profit maximisation problem, $g$ considers values for $k \in\left[k_{\min }=1-3 s, k_{\max }=1+3 s\right]$.

\section{Results}

We begin with confirming that, for several general economic properties, the market model behaves as expected in reality. First, $g$ makes profits with both formats and across all settings. Profits also correlate with settings like one would expect. They are positively correlated to changes in $R_{g}, D_{\max }^{A}$ and $\rho_{\min }^{A}$ and negatively correlated to $r_{m}, \alpha^{A}$ and $\beta^{A}$. Second, $g$ 's presence increases competition as he can offer electricity below market price. We simulated the market without $g$ (thus decreasing the number of suppliers by one). As should be expected, the aggregated unit price is significantly higher than with $g$ 's presence. Finally, in comparison to the oligopolistic scenario, the competitive scenario has lower aggregated market prices, as well as market power and profits for $g$.

We now turn to three major observations, concerning notable differences or similarities in outcomes when $g$ uses either the $B E N C H$ or the $U N I$ format:

Observation 1: The $U N I$ format substantially reduces market prices and market power in exploitable settings. In the default settings, the $U N I$ and $B E N C H$ format show no significant ${ }^{1}$ differences in market power. The biggest opportunities for $g$ to exercise market power exist in settings with larger values for $\rho_{m i n}^{A}$, because then the difference between offer prices of $S_{-g}$ and $g$ 's costs is high and $g$ can thus increase his profit margin. The settings in which $\rho_{\text {min }}^{A} \geq 24$ show by far the highest aggregated market prices, as well as market power and profits for $g$. The differences in market power between the $U N I$ and the $B E N C H$ format are significant ${ }^{1}$, with the exception of the baseline scenario where $r_{m}=0.1$. The results show that $g$ exploits this opportunity less when he uses the $U N I$ format.

Specifically, $g$ is lowering the price $\rho_{g}^{A}$ on market $A$, and as a result the aggregated market prices are lower than with the $B E N C H$ format (note that the most quantity is sold on market $A$ and thus lowering $\rho_{g}^{A}$ has strong effects). See Figure 2 for the most substantial case, where the presence of $g$ when using the $U N I$ format has an impact on aggregated market prices up to 2.7 times as when $g$ uses the $B E N C H$ format. The results also show a clear reduction in market power. In the default settings $\left(\rho_{\min }^{A}=20.68\right), g$ has more market power with the $U N I$ format. However, in settings with $\rho_{\min }^{A} \geq 24, g$ gains substantially less market power with respect to the default setting when using the $U N I$ format and therefore has less market power than with the $B E N C H$ format. See Figure 3 for the most substantial case, where the $U N I$ format has up to $11 \%$ less market power.

Observation 2: The $U N I$ format is stable against noise. In the oligopolistic scenario, $g$ has substantial market power due to his position in this less competitive market setting. If $g$ can be less certain about market outcomes due to increasing noise (note that we do not model noise for other

\footnotetext{
${ }^{1}$ We performed Student's T-Tests and tested for $p \leq 0.01$.
} 


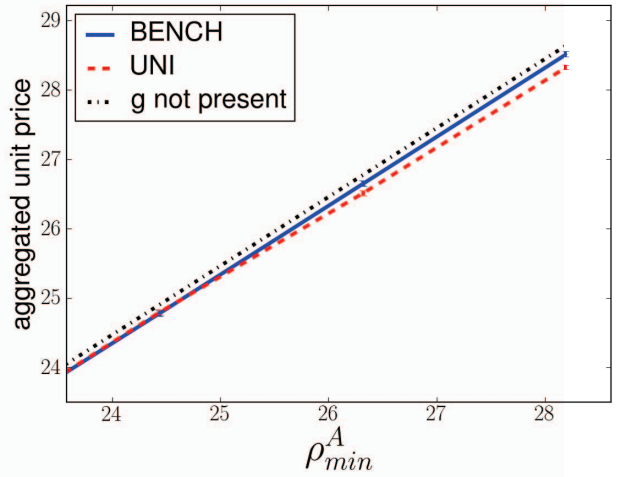

Figure 2. Aggregated unit price against increasing competition prices (competitive scenario, $r_{m}=0.3$ ). Results shown with ${ }_{-}^{+} 1$ standard deviation. The dotted line indicates the default setting for $\phi$.

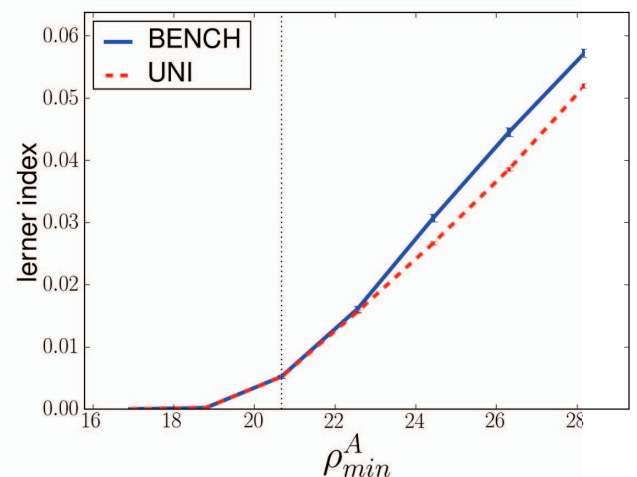

Figure 3. Market power against increasing competition prices (competitive scenario, $r_{m}=0.3$ ). Note the scale of the y-axis.

market participants), his market power decreases when he is using the $B E N C H$ format, but stays constant (on average) with the $U N I$ format (see Figure 4 for an example).

Observation 3: In all other settings, the $U N I$ format has comparable impacts on overall market outcomes. Although the formats are constructed differently and show distinct bidding behaviour (in the simulated settings not covered in observation 1 and 2, the $U N I$ format sells less $Q_{g}^{B}$ at a higher price $\rho_{g}^{B}$, see Figure 5 for an example), the impact of $g$ on market prices does not differ significantly ${ }^{1}$ when $g$ uses the $U N I$ or $B E N C H$ format (if noise is at least as high as in the default setting). From the perspective of the market, the $U N I$ format thus shows no substantial drawback in these cases.

\section{E. Discussion and future work}

Let us first consider why the $B E N C H$ format bids lower prices on market $B$ (see observation 3 and Figure 5). Given his bid $b_{g}^{A}$ on market $A, g$ searches for the bid $b_{g}^{B}$

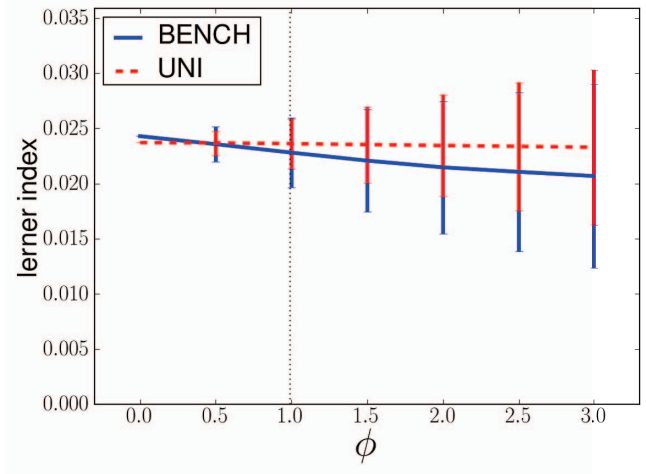

Figure 4. Market power against increasing uncertainty of $g$ about market outcomes (oligopolistic scenario, $r_{m}=0.3$ ).

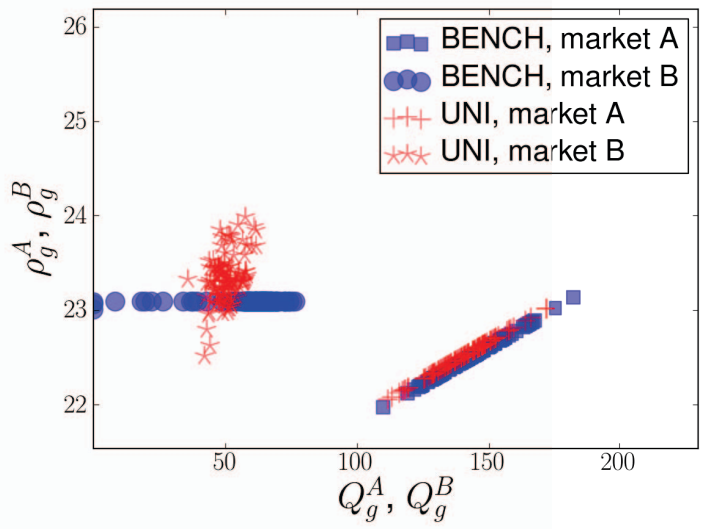

Figure 5. Sampled outcomes for $g$ (oligopolistic scenario, $r_{m}=0.3$ ).

that maximises the expected profits. Because $b_{g}^{B}$ reflects a constant price $\rho_{g}^{B *}$, an unexpectedly low residual demand function (a result of a low value for $k^{B}$ ) can decrease $Q_{g}^{B}$ dramatically (especially when the slope of $D_{g}^{B}$ is high). Thus, the $B E N C H$ format bids a lower value for $\rho_{g}^{B *}$ to stabilise the expected amount $Q_{g}^{B}$. Specifically, consider a deterministic setting $\left(k^{A}=k^{B}=1\right): g$ maximises profits by selling $Q_{g}^{B}=Q_{g}^{o p t}$ at price $\rho$, where $D_{g}^{B}\left(\rho, k^{B}\right)=Q_{q}^{o p t}$ for $k^{B}=1$. However, in a non-deterministic setting, the $B E N C H$ format bids a price $\rho_{g}^{B *}$ lower than $\rho$. This also explains observation 2: The higher the uncertainty about market outcomes, the lower $g$ will bid on market $B$ when using the $B E N C H$ format. As the $U N I$ format is bidding a function instead of a constant price, it does not face this problem and has stable market power. This makes the $U N I$ format appealing to bidders, as it is less vulnerable to increasing uncertainty about market outcomes.

We now turn to observation 1 and discuss bidding be- 
haviour under exploitable market settings (here modelled by large values for $\rho_{m i n}^{A}$ ). In most settings we studied, multiple near-optimal combinations of quantities and prices exist. Though the $U N I$ format is less flexible than the $B E N C H$ format (because bids $b_{g}^{A}$ and $b_{g}^{B}$ are based on one bid $b_{g}$ ), it is likely to find a bid $b_{g}$ that realises one of them, as is evident in the good performance across all settings. However, the market settings in question (with $\rho_{\text {min }}^{A} \geq 24$ ) are so favourable for $g$ that he can sell all capacity on both markets $\left(Q_{g}^{A}=Q_{g}^{U}\left(1-r_{m}\right)\right.$ and $\left.Q_{g}^{B}=Q_{g}^{\text {opt }}\right)$. This means that there exists only one pair of optimal quantities $\left(Q_{g}^{U}\left(1-r_{m}\right)\right.$ and $\left.Q_{g}^{o p t}\right)$ and the optimisation problem is reduced to finding the optimal prices. However, if $Q_{g}^{A}$ is fixed, the distance between bids $b_{g}^{A}$ and $b_{g}^{B}$ with regard to the price axis is fixed as well (because the minimum price of $b_{g}^{B}$ is $c_{g}^{*}+2 R_{g} Q_{g}^{A}$, see (9)). Thus, it becomes highly unlikely that $g$ can bid optimal prices in both markets. We conclude that under very favourable conditions for $g$, the $U N I$ format restricts $g$ from realising the full potential market power. As a result, the $U N I$ format lowers bid $b_{g}^{A}$ substantially in order to not overprice on market $B$. This makes the $U N I$ format appealing to market makers, as it restricts the exercise of excessive market power in exploitable market settings.

The decision-theoretic approach taken in this work gives first insights into this complex problem setting. However, future work could further evaluate the $U N I$ format in a multi-agent setting. By including the decision-making of multiple actors, the social efficiency improvements can be studied in more detail. In addition, certain assumptions may be loosened. First, individual ratios $r_{g}$ per bidder would model a more dynamic market setting. Each bidder would decide how much flexibility he actually can offer, depending on his portfolio as well as adaptive strategies, where for example $r_{g}=0$ means that $g$ is not taking part in market $B$. If suppliers bid on several $r_{g}$ values, the $S O$ can increase market efficiency by choosing among them (see also [5]). Second, bidders can be allowed to adjust both bid parameters (see Section III-A and one might investigate the usefulness of strategies that rely on this. Finally, it is important to consider the bid format for the demand side, as well. In a companion work (currently under review), which is more tailored to the electrical engineering community, we model a flexible consumer and formulate the problem such that he bids a demand bid on market $A$ and a supply bid on market $B$, offering to reduce his planned consumption.

\section{CONCLUSION}

This work analyses the decision problem of a smart bidding agent in a two-settlement electricity auction. It provides a parametrised, stochastic market model with discriminatory bids. The profit maximisation problem of a generator is formalised and studied with Monte-Carlo simulations and careful parameter analysis. We model the current stateof-the-art bid format and incorporate a novel format that enables simpler computation by agents through unification of the two bids. Experimental simulations show that the unified format restricts market power of suppliers in exploitable market settings, and is also less vulnerable to increasing uncertainty of bidders about market outcomes.

\section{REFERENCES}

[1] R. Baldick. Electricity market equilibrium models: the effect of parametrization. IEEE Transactions on Power Systems, 17(4):1170-1176, Nov. 2002.

[2] D. Damianov, J. Oechssler, and J. Becker. Uniform vs. discriminatory auctions with variable supply-experimental evidence. Games and Economic Behavior, 68(1):60-76, 2010.

[3] N. Fabra, N. Von Der Fehr, and D. Harbord. Designing electricity auctions: uniform, discriminatory and vickrey. Technical report, Universidad Carlos III de Madrid and University of Oslo, 2002.

[4] N. Höning and H. La Poutré. Flexible Consumers Reserving Electricity and Offering Profitable Downward Regulation. In Third IEEE PES Conference On Innovative Smart Grid Technologies (ISGT), 2012.

[5] N. Höning, H. Noot, and H. La Poutré. Integrating power and reserve trade in electricity networks [extended abstract]. In Proceedings of the 10th International Conference on Autonomous Agents and Multiagent Systems (AAMAS), 2011.

[6] P. Klemperer and M. Meyer. Supply function equilibria in oligopoly under uncertainty. Econometrica, 57(6):1243-1277, 1989.

[7] V. Krishna. Auction Theory. Academic Press, San Diego, 2002.

[8] R. Lafferty et al. Demand responsiveness in electricity markets. Technical report, Federal Energy Regulatory Commission, 2001.

[9] A. Mas-Collel et al. Microeconomics. Oxford University Press, 1995.

[10] J. Nelder and R. Mead. A simplex method for function minimization. The computer journal, 7(4):308, 1965.

[11] S. Oren. Design of ancillary service markets. Proceedings of the 34th International Conference on System Sciences, 2000.

[12] J. Perloff. Microeconomics: Theory and Applications with Calculus. Addison Wesley, 2007.

[13] S. Stoft. Power Market Economics - Designing Markets For Electricity. Wiley-Interscience, 2002.

[14] J. Sun and L. Tesfatsion. Dynamic Testing of Wholesale Power Market Designs: An Open-Source Agent-Based Framework. Computational Economics, 30(3):291-327, Aug. 2007.

[15] A. Virag, A. Jokic, R. Hermans, and P. van Den Bosch. Combined Bidding at Power and Ancillary Service Markets. In Proceedings of the 8th International Conference on the European Energy Market, Zagreb, 2011. 